\title{
Glucose intolerance develops prior to increased adiposity and accelerated cessation of estrous cyclicity in female growth-restricted rats
}

\author{
Suttira Intapad ${ }^{1-4}$, John Henry Dasinger ${ }^{1-4}$, Andrew D. Brown' ${ }^{1}$, Joel M. Fahling ${ }^{1}$, Joyee Esters ${ }^{1}$ and Barbara T. Alexander ${ }^{1-4}$
}

BACKGROUND: The incidence of metabolic disease increases in early menopause. Low birth weight influences the age at menopause. Thus, this study tested the hypothesis that intrauterine growth restriction programs early reproductive aging and impaired glucose homeostasis in female rats.

METHODS: Estrous cyclicity, body composition, and glucose homeostasis were determined in female control and growth-restricted rats at 6 and 12 mo of age; sex steroids at 12 mo. RESULTS: Glucose intolerance was present at 6 mo of age prior to cessation of estrous cyclicity and increased adiposity in female growth-restricted rats. However, female growth-restricted rats exhibited persistent estrus and a significant increase in adiposity, fasting glucose, and testosterone at $12 \mathrm{mo}$ of age $(P<0.05)$. Insulin release in response to a glucose challenge was blunted in conjunction with a reduction in protein expression of pancreatic glucose transporter type 2 and estrogen receptor- $\alpha$ at 12 mo of age in female growth-restricted rats $(P<0.05)$.

CONCLUSION: This study demonstrated that slow fetal growth programmed glucose intolerance that developed prior to early estrous acyclicity; yet, fasting glucose levels were elevated in conjunction with increased adiposity, accelerated cessation of estrous cyclicity and a shift toward testosterone excess at 12 mo of age in female growth-restricted rats.

A ge at menopause usually occurs from 50 to $55 \mathrm{y}$ of age (1). However, women exposed to famine during late gestation (2) or born low birth weight $(3,4)$ exhibit a greater prevalence of early menopause. Experimental studies indicate that prenatal exposure to maternal undernutrition programs markers of early reproductive senescence (5-8). Our laboratory utilizes a model of intrauterine growth restriction (IUGR) induced via placental insufficiency initiated at day 14 of gestation in the Sprague Dawley (SD) rat (9). Female growth-restricted offspring are normotensive at 6 mo of age but develop hypertension and an increase in total fat mass by 12 mo of age (10). Reproductive senescence and cessation of estrous cyclicity occur about 18 mo of age in female SD rats (11). We previously reported that uterine weight is similar in female growth-restricted rats relative to female control that exhibit hypertension at 12 mo of age (10). However, this study did not determine estrous cyclicity or hormone levels in conjunction with measure of blood pressure. Therefore, based on studies implicating that undernutrition during fetal life programs early reproductive aging, one goal of this study was to test the hypothesis that early reproductive senescence coincides with the development of increased adiposity and hypertension in female growth-restricted offspring.

Menopause is associated with changes in the hormonal milieu that results in a shift in the balance of estrogen and testosterone with a transition toward a testosterone dominated milieu (12). Testosterone is positively associated with visceral fat (12) and blood pressure (13) in postmenopausal women. In addition, increased testosterone and adiposity predict the risk for type 2 diabetes (T2D) after menopause (14). Early onset menopause increases the risk of T2D (15) and the age-adjusted risk for T2D is increased in low-birth-weight (LBW) women (16). Yet, the combined effect of birth weight and early reproductive aging on metabolic health is not known. Experimental models demonstrate that placental insufficiency or maternal nutrient restriction during fetal life programs impaired glucose and insulin homeostasis (17) in a manner that is sex-specific in young adulthood $(18,19)$. Age exacerbates the developmental programming of hyperinsulinemia in female offspring exposed to maternal protein restriction during fetal life (20). Thus, this study also tested the hypothesis that placental insufficiency programs impaired glucose homeostasis in the female growth-restricted rat that precedes or is augmented in conjunction with estrous acyclicity and a change in the estradiol to testosterone ratio.

\section{RESULTS}

Effect of Intrauterine Growth Restriction on Reproductive Aging Previously, we reported that uterine weight did not differ in growth-restricted rats relative to age-matched control

\footnotetext{
'Department of Physiology and Biophysics, University of Mississippi Medical Center, Jackson, Mississippi; ${ }^{2}$ Women's Health Research Center, University of Mississippi Medical Center, Jackson, Mississippi; ${ }^{3}$ Center for Cardiovascular and Renal Research, University of Mississippi Medical Center, Jackson, Mississippi; ${ }^{4}$ Mississippi Center for Obesity Research, University of Mississippi Medical Center, Jackson, Mississippi. Correspondence: Barbara T. Alexander (balexander@umc.edu) 
counterparts at 12 mo of age (10). To determine if sex steroids differed in normotensive female control rats relative to female growth-restricted rats that demonstrated a significant increase in blood pressure at 12 mo of age, samples collected from this previous study were analyzed for circulating estradiol, testosterone, luteinizing hormone, or follicle-stimulating hormone levels (10). Estradiol levels did not differ at 12 mo of age upon comparison of growth-restricted rats relative to control (Figure 1, panel a). However, circulating testosterone levels were significantly elevated in the growth-restricted rats compared to control at 12 mo of age (Figure 1, panel b). This resulted in a shift in the testosterone to estradiol balance in growth-restricted rats (Figure 1, panel c). Follicle-stimulating hormone and luteinizing hormone levels did not differ (Figure 1, panels $\mathrm{d}$ and e).

Based on these findings, we assessed estrous cyclicity in another group of animals. In this group, control and growth-restricted rats demonstrated no significant difference in cycle length or the percentage of each stage of the estrus cycle (metaestrus, diestrus, proeastrus, or estrus) (Figure 1, panel f). By 11 mo of age and continuing up to $12 \mathrm{mo}$ of age control rats exhibited elongation of the estrus cycle but no significant difference in the percent distribution in each stage of the estrus cycle (Figure 1, panel g). However, by 11 mo of age growth-restricted rats were primarily in persistent estrus (Figure 1, panel g) which continued for $30 \mathrm{~d}$. Estradiol and testosterone in this group reflected levels reported in Figure 1, panels a and b. Evaluation of ovarian morphology and follicle number will be needed to corroborate accelerated aging of the reproductive system in this model of IUGR.

\section{Effect of Intrauterine Growth Restriction on Body Composition} Birth weight was significantly reduced $(P<0.001)$ in growth-restricted offspring compared to control (Table 1).
Growth-restricted rats exhibited catch-up growth by 2 mo of age with no difference in body weight noted at 6 or 12 mo of age relative to age-matched controls (Table 1). No difference in total body fat mass was observed at 6 mo of age in growth-restricted relative to control (Table 1). However, total body fat mass was significantly increased in growth-restricted $(P<0.05)$ relative to control at 12 mo of age (Table 1$)$ when total lean mass and total body water content were significantly reduced in growth-restricted rats $(P<0.05)$ (Table 1$)$; observations consistent with those previously reported for this model (10). Visceral fat mass did not differ at 6 mo of age but was elevated by 12 mo of age in control and growth-restricted rats $(P<0.05$ vs. 6 mo of age counterpart). However, the magnitude of this increase was significantly greater in growth-restricted relative to age-matched control (Table 1); an observation also noted previously (10). Growth-restricted rats showed a slightly lower water intake independent of age. Overall, food intake, water intake, and urinary excretion did not change with age (Table 1).

\section{Effect of Intrauterine Growth Restriction on Glucose Homeostasis}

To determine if IUGR programs glucose intolerance prior to the development of estrous acyclicity, we examined glucose tolerance at 6 and 12 mo of age. Growth-restricted rats at 6 mo of age exhibited a significant elevation in blood glucose at $30 \mathrm{~min}$ postglucose challenge in the fasted state when compared to age-matched control (Figure 2, panel a). Yet, at 12 mo of age glucose remained significantly elevated up to $120 \mathrm{~min}$ postglucose challenge in growthrestricted rats relative to control (Figure 2, panel a). The area under the curve (AUC) for the response to glucose
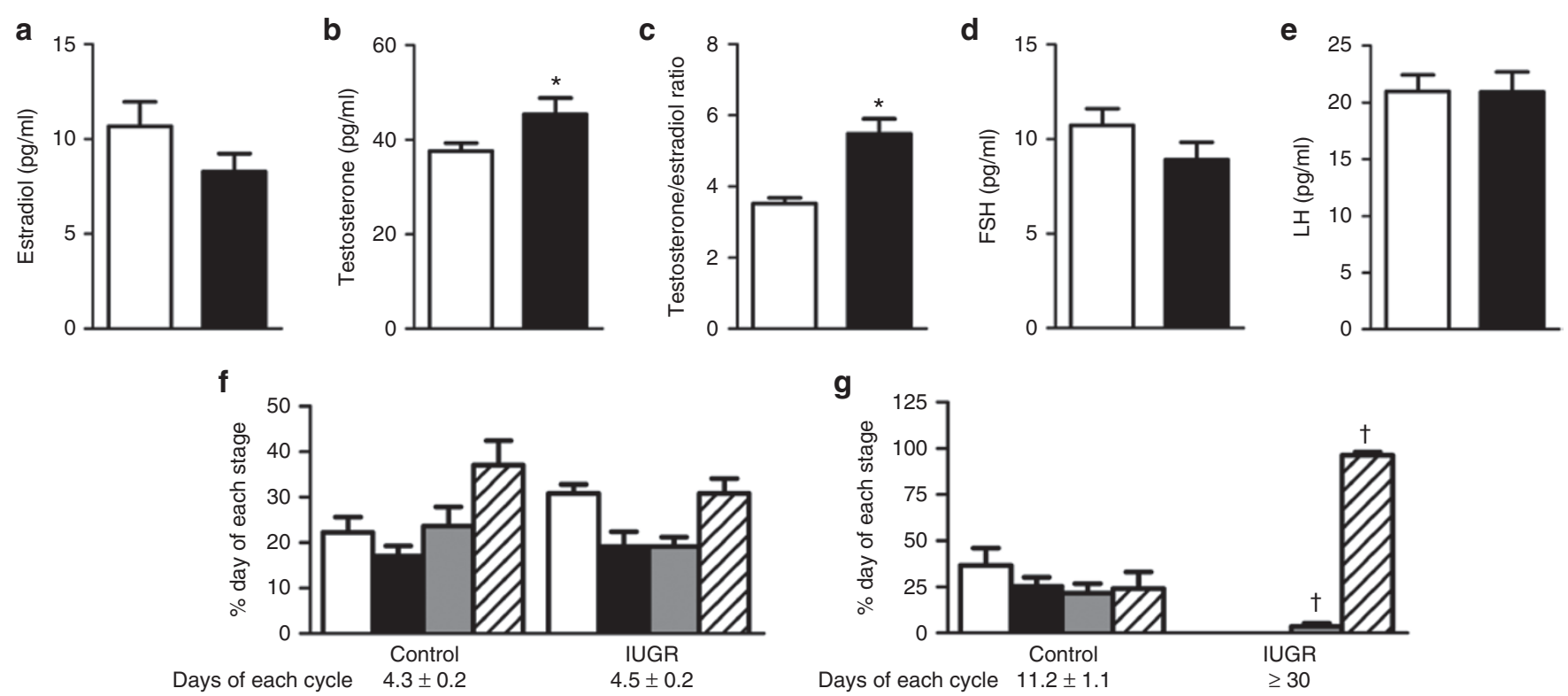

Figure 1. Hormone levels and estrous cyclicity. Serum estradiol (a), testosterone levels (b), testosterone/estradiol ratio (c), follicle-stimulating hormone (d), and LH (e) in control and intrauterine growth restriction (IUGR) at 12 mo of age. Values are mean \pm SEM ( $n=13$ per group). ${ }^{*} P<0.05$ vs. control (white columns) and IUGR (black columns) using unpaired $t$-test. Percent of each stage in estrous cycle and number of days per cycle at 6 (f) and 12 (g) months of age: metestrus (white columns), diestrus (black columns), proestrus (gray columns), and estrus (hatched columns). ${ }^{\dagger} P<0.05$ represents the comparison between each stage of estrus cycle of age-matched control and IUGR. Values are the mean \pm SEM ( $n=6$ per group). 


\section{Articles | Intapad et al.}

Table 1. Body composition; food and water intake

\begin{tabular}{|c|c|c|c|c|c|c|c|}
\hline \multirow[b]{2}{*}{ Parameters } & \multicolumn{2}{|c|}{ Six months } & \multicolumn{2}{|c|}{ Twelve months } & \multicolumn{3}{|c|}{ Two-way ANOVA } \\
\hline & Control & IUGR & Control & IUGR & IUGR & Age & Interaction \\
\hline Birth weight (g) & $6.14 \pm 0.03$ & $4.99 \pm 0.25^{*}$ & $6.43 \pm 0.28$ & $4.55 \pm 0.20^{*}$ & - & - & - \\
\hline Total body fat (g)/BW (g) & $0.091 \pm 0.009$ & $0.087 \pm 0.006$ & $0.104 \pm 0.006$ & $0.142 \pm 0.015^{t, \ddagger}$ & ns & $P<0.01$ & ns \\
\hline Total lean mass (g)/BW (g) & $0.854 \pm 0.008$ & $0.865 \pm 0.017$ & $0.786 \pm 0.028$ & $0.689 \pm 0.029^{t, \neq}$ & ns & $P<0.01$ & ns \\
\hline Visceral fat (g)/BW (kg) & $2.12 \pm 0.4$ & $3.08 \pm 1.1$ & $11.2 \pm 1.8^{\dagger}$ & $23.6 \pm 4.7^{t, \neq}$ & $P<0.001$ & $P<0.01$ & $P<0.05$ \\
\hline Food intake (g)/BW (g)/day & $0.064 \pm 0.008$ & $0.042 \pm 0.009$ & $0.045 \pm 0.006$ & $0.041 \pm 0.006$ & ns & ns & ns \\
\hline Water intake (g)/BW (g)/day & $0.112 \pm 0.008$ & $0.075 \pm 0.021$ & $0.104 \pm 0.009$ & $0.074 \pm 0.009$ & $P<0.05$ & ns & ns \\
\hline Twenty-four hours urinary excretion (ml) & $12.8 \pm 1.2$ & $14.4 \pm 3.1$ & $20.5 \pm 2.3$ & $13.6 \pm 1.7$ & ns & ns & ns \\
\hline \multicolumn{8}{|c|}{$\begin{array}{l}\text { Values are the mean } \pm \text { SEM, }(n=14 \text { rats per group). } \\
\text { IUGR, intrauterine growth restriction. } \\
{ }^{*} P<0.05 \text { vs. control using unpaired } t \text {-test. } P \text { values the significance in the effect for the respective source of variation (IUGR or age) using two-way ANOVA. }{ }^{+} P<0.05 \text { in Bonferroni post } \\
\text { hoc test comparing age-matched control and IUGR offspring. }{ }^{P} P<0.05 \text { Bonferroni post hoc test comparing same group in different age. }\end{array}$} \\
\hline
\end{tabular}

load was significantly increased in growth-restricted rats at 6 and 12 mo of age (Figure 2, panel b) indicating that birth weight was inversely correlated to AUC. AUC was also significantly greater in growth-restricted rats at $12 \mathrm{mo}$ of age relative to 6-mo counterparts denoting a significant interaction with age in growth-restricted rats. No difference was noted in the overnight fasted blood glucose concentration in growth-restricted compared to age-matched control rats at 6 mo of age. However, overnight fasted blood glucose concentrations were significantly increased with age in growth-restricted rats $(P<0.05)$ (Figure 2 , panel $c)$. Insulin tolerance was similar in growth-restricted relative to agematched control at 6 and 12 mo of age (Figure 2, panels $d$ and e). Fasting plasma insulin levels did not differ at 6 or 12 mo of age (Figure 2, panel f) and the HOMA index was not different between groups at either age (control: $0.843 \pm 0.12$ and $0.556 \pm 0.16$, IUGR: $0.889 \pm 0.13$ and $0.788 \pm 0.17 ; 6$ and 12 mo of age, respectively). Glucose-induced insulin release was significantly blunted at $30 \mathrm{~min}$ postglucose challenge in growth-restricted rats relative to age-matched control at 12 mo of age (Figure 3, panel a). As previously reported (10), plasma leptin levels were similar at 6 mo of age, but were significantly increased in growth-restricted offspring at 12 mo of age relative to age-matched control (10).

\section{Effect of Intrauterine Growth Restriction on Protein Expression of GLUT2, GLUT4, IR $\beta$, and ER $\alpha$ in Insulin-Sensitive Tissues}

At 12 mo of age, pancreas weight did not differ between control and growth-restricted rats $(5.31 \pm 0.21 \mathrm{vs} .5 .26 \pm 0.51 \mathrm{~g} / \mathrm{kg}$ BW; control vs. IUGR, respectively). However, insulin release in response to a glucose challenge in the fasted state was significantly reduced in growth-restricted compared to control (Figure 3, panel a). Protein expression of pancreatic glucose transporter type 2 (GLUT2), a glucose sensor, was significantly decreased in growth-restricted relative to control rats at $12 \mathrm{mo}$ of age (Figure 3, panel b). Protein expression of estrogen receptor- $\alpha(\mathrm{ER} \alpha)$, which is reported to play an important role in the regulation of insulin biosynthesis and insulin secretion in the pancreas (21) was significantly reduced in pancreatic tissue of growth-restricted rats relative to control at 12 mo of age (Figure 3, panel c). Protein expression for GLUT4 did not differ in muscle (Figure 3, panel d) but was significantly reduced in white adipose tissue from growth-restricted relative to control (Figure 3, panel f). Protein expression of insulin receptor- $\beta$ (IR $\beta$ ) also did not differ in muscle (Figure 3, panel e); yet, IR $\beta$ protein expression was significantly upregulated in white adipose tissue of growth-restricted relative to control rats at 12 mo of age (Figure 3, panel g).

\section{Effect of Ovariectomy on Glucose Tolerance, Body Composition, Testosterone, Blood Pressure and Uterine Weight in Control, and Growth-Restricted Offspring}

Ovariectomy (OVX) at $10 \mathrm{wk}$ of age significantly increased the glucose concentration at $30 \mathrm{~min}$ postglucose challenge in control rats at 6 mo of age $(P<0.001)$ (Figure 4 , panel a) resulting in an increase in the AUC (Figure 4, panel c). At 12 mo of age ovariectomy did not significantly alter the glucose response to a glucose challenge in the fasted state or AUC in control relative to intact counterparts (Figure 4, panels b and d). Ovariectomy did not alter AUC in growth restricted rats at 12 mo of age relative to intact counterparts (Figure 4, panel d). Yet, the pattern for glucose concentration postglucose challenge differed following ovariectomy in growth-restricted rats at 12 mo of age relative to intact counterparts although glucose remained significantly elevated at $120 \mathrm{~min}$ postglucose challenge as observed in intact counterparts (Figure 4, panel b). Ovariectomy had no significant effect on fasting blood glucose in growth-restricted rats relative to intact counterparts at 12 mo of age (Figure 4, panel f). Fat mass was increased by ovariectomy in control and growth-restricted rats at 6 (Figure 4, panel g) and 12 mo of age relative to their intact counterparts; ovariectomy also significantly increased fat mass in growth-restricted rats relative to control counterpart at 12 mo of age (Figure 4, panel h). 

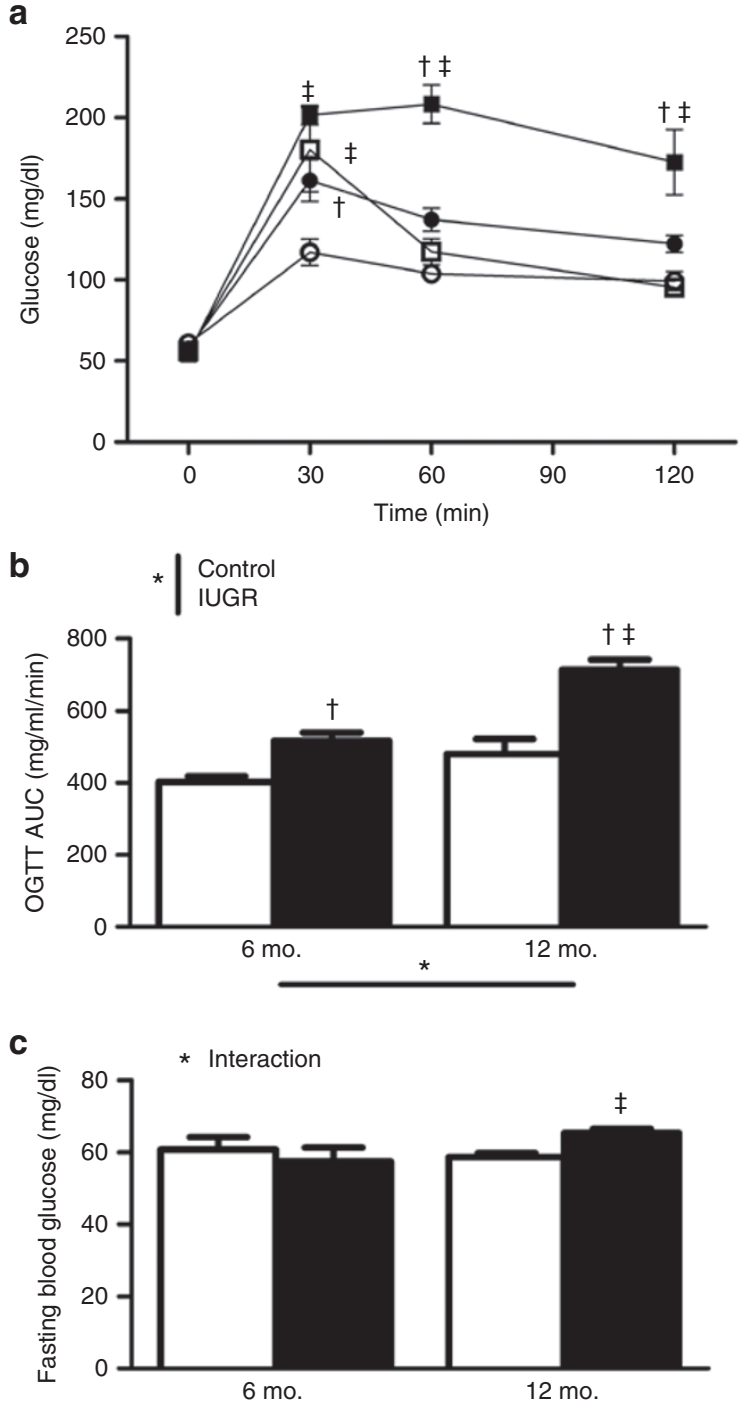

d

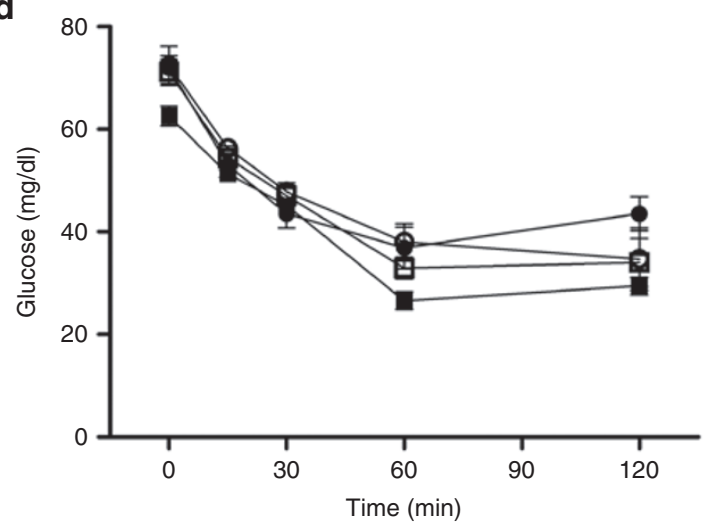

e

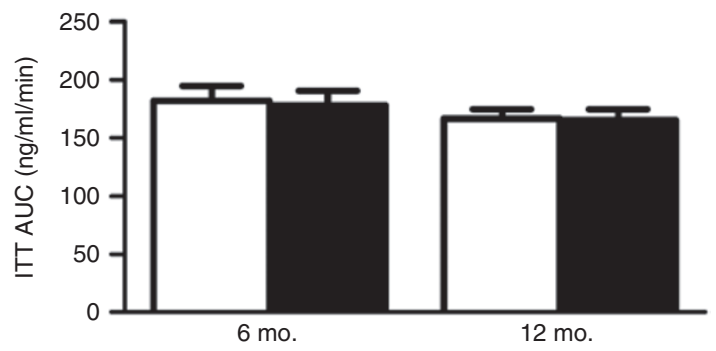

$\mathbf{f}$

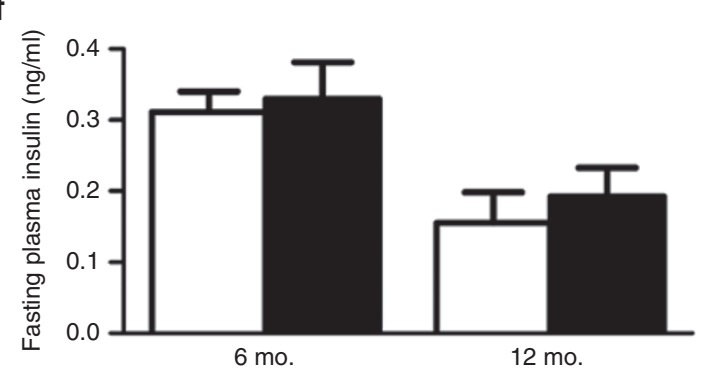

Figure 2. Glucose homeostasis. Oral glucose tolerance test (OGTT) (a) and insulin tolerance test (ITT) (b) in control and intrauterine growth restriction (IUGR) at 6 and 12 mo of age. For OGTT: area under the curve (AUC) (b) and fasting blood glucose levels (c). For ITT: area under the curve (AUC) (e) and fasting plasma insulin level (f). For (a) and (d): open circle (control $6 \mathrm{mo}$ ), closed circle (IUGR $6 \mathrm{mo}$ ), open rectangle (control $12 \mathrm{mo}$ ), and closed rectangle (IUGR 12 mo). For (b), (c), (e), and (f): white columns (control) and black columns (IUGR). ${ }^{*} P$ values next to each label represent significance $(P<0.05)$ (IUGR or age or interaction) using two-way ANOVA; ${ }^{+} P<0.05$ represents IUGR vs. control at the same age; ${ }^{\ddagger} P<0.05$ represents comparison vs. 6 mo of age between similar group using Bonferroni post hoc test, $(n=6-9$ per group).

Uterine weight was significantly decreased at 12 mo of age following ovariectomy at $10 \mathrm{wk}$ of age in control and growthrestricted rats (control: $0.38 \pm 0.06$ vs. $0.08 \pm 0.01$ and IUGR: $0.25 \pm 0.05$ vs. $0.10 \pm 0.02 \mathrm{~g} / \mathrm{kg} \mathrm{BW}$, intact vs. OVX; respectively; $P<0.05$, intact vs. OVX counterpart). Ovariectomy did not further increase testosterone levels in growth-restricted relative to intact counterparts at 12 mo of age $(49.7 \pm 6.1$ vs. $48.8 \pm 5.4 \mathrm{pg} / \mathrm{ml}$; OVX IUGR vs. intact IUGR; respectively), but ovariectomy did increase testosterone levels in intact control relative to ovariectomized control at $12 \mathrm{mo}$ of age $(39.7 \pm 1.8$ vs. $48.6 \pm 2.6 \mathrm{pg} / \mathrm{ml} ; P<0.05$, intact vs. OVX, respectively). Testosterone levels did not differ in ovariectomized growthrestricted relative to ovariectomized female control or intact female growth-restricted rats.

\section{DISCUSSION}

This study noted several findings of novel importance. Circulating testosterone levels were increased at $12 \mathrm{mo}$ of age in growth-restricted rats previously reported to demonstrate increased visceral adiposity and hypertension. IUGR also programmed an increase in testosterone levels in female growth-restricted rats that exhibited persistent estrous at 11 mo of age that persisted to $12 \mathrm{mo}$. Glucose intolerance but not an increase in fasting blood glucose levels was observed in growth-restricted rats at 6 mo of age or prior to the development of increased total fat mass and estrous acyclicity. However, fasting blood glucose levels in growth-restricted rats were increased at 12 mo of age. Glucose-stimulated insulin release was blunted in response to an oral glucose 


\section{Articles | Intapad et al.}
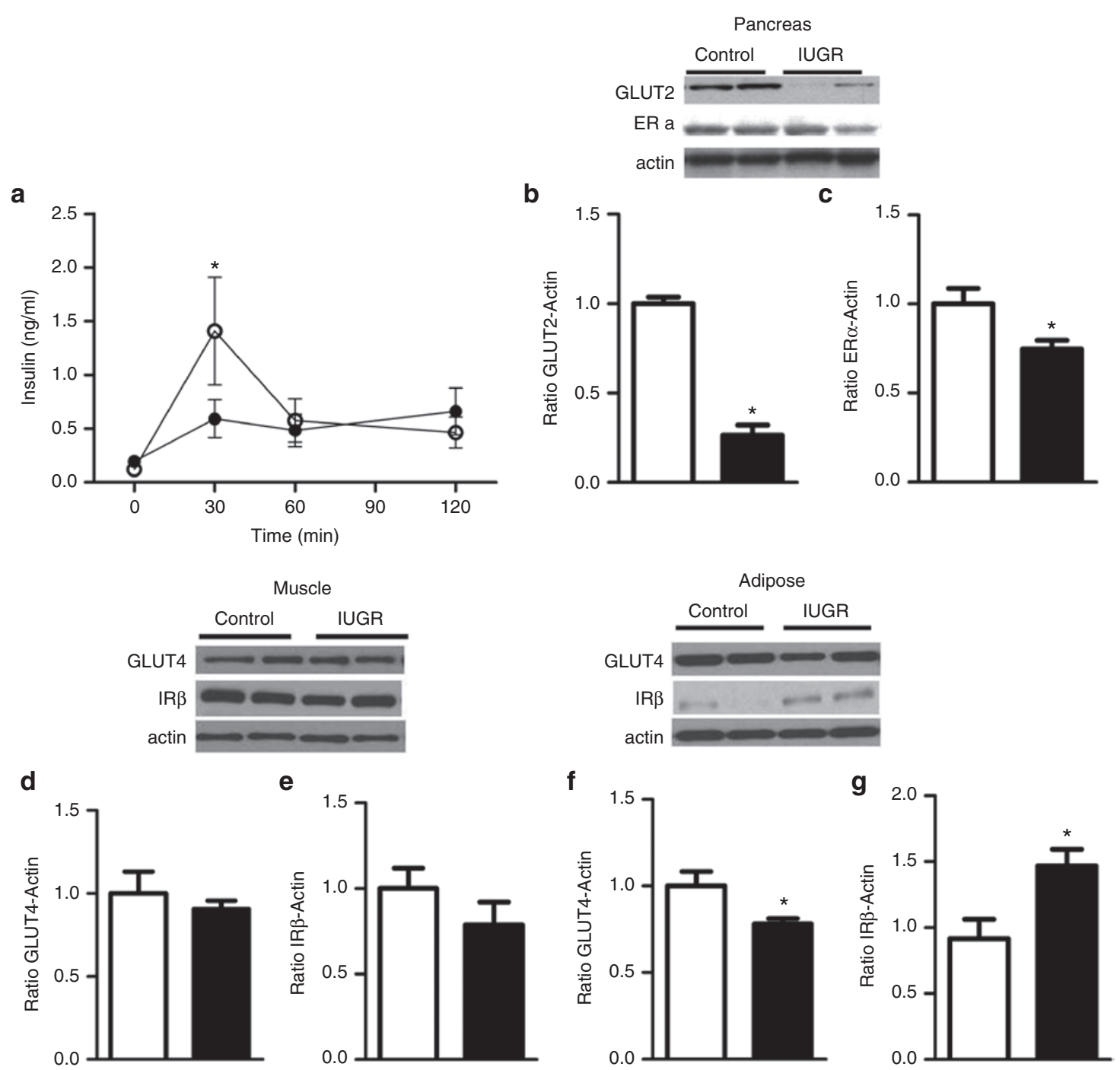

Figure 3. Insulin release; protein expression in insulin-sensitive tissues. Glucose-stimulated insulin release in response to an oral glucose challenge in fasted state in control and intrauterine growth restriction (IUGR) at 12 mo of age (a). Protein expression of glucose transporter type 2 (GLUT-2) and estrogen-receptor (ER- $\alpha$ ) in pancreas adjusted to actin (b), protein expression of glucose transporter type 4 (GLUT-4) and insulin receptor- $\beta$ (IR $\beta$ ) adjusted to actin in gastrocnemius muscle (c); protein expression of GLUT- 4 and IR $\beta$ adjusted to actin in dorsal white adipose tissue (d). Shown are two representative samples. ${ }^{*} P<0.05$ vs. control, ( $n=4-6$ per group) using unpaired $t$-test. For (a): open circle (control 12 mo) and closed circle (IUGR 12 mo). For (b), (c), (d), (e), and (f): columns (control) and black columns (IUGR). All samples were derived at the same time and processed in parallel.

challenge in growth-restricted rats at 12 mo of age. Pancreatic ER $\alpha$, GLUT2, and adipose GLUT4 protein expression were also significantly reduced in growth-restricted relative to agematched control at 12 mo of age. In addition, ovariectomy induced glucose intolerance, increased the AUC for the oral glucose tolerance test (OGTT), elevated fasting blood glucose levels, and increased fat mass in control rats at 6 mo of age. Ovariectomy also increased fat mass and fasting glucose levels in growth-restricted rats relative to intact counterparts at 6 mo of age without an additive effect on AUC or change in the pattern of the OGTT. Ovariectomy further increased fat mass in control rats at 12 mo of age with no effect on glucose homeostasis. Ovariectomy increased fat mass in growthrestricted rats at $12 \mathrm{mo}$ of age relative to ovariectomized control or intact growth-restricted counterparts but did not alter AUC for the OGTT or fasting blood glucose levels.
Circulating testosterone levels were increased in female growth-restricted rats that were previously reported to exhibit increased adiposity and hypertension at 12 mo of age (10). Since testosterone is positively coincident with hypertension and visceral adiposity in postmenopausal women $(12,13)$, we assessed estrous cyclicity and sex steroids in another group of animals. The normal pattern of estrous cyclicity in the rat is usually $4-5 \mathrm{~d}$ in length and contains a normal interval distribution of metaestrus, diestrus, proeastrus, and estrus stages after puberty (22). Cycle length increases in the rat with a transition to persistent estrus, an acyclic state characterized by elevated estradiol (22) that occurs around 18 mo of age in the SD rat (11). In this study group, female growth-restricted rats exhibited persistent estrous as early as 11 mo of age that persisted to $12 \mathrm{mo}$ of age when testosterone was significantly increased but estradiol levels were not altered. Perimenopause in women 
a

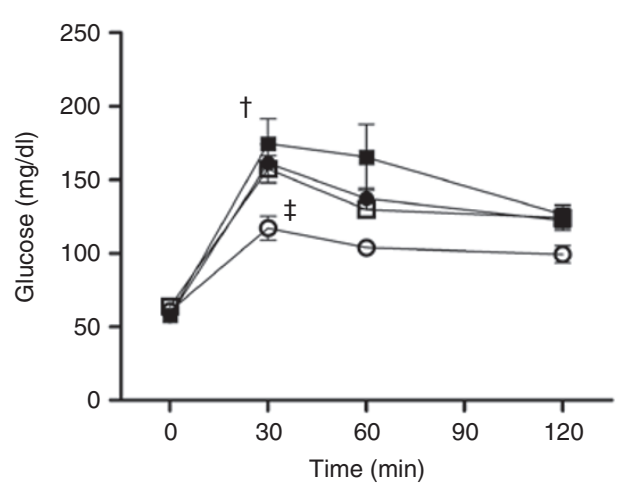

C

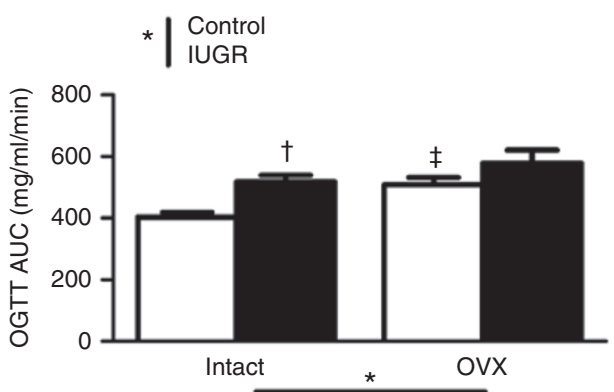

e

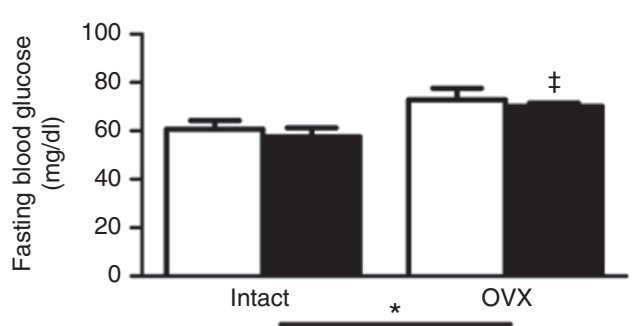

g

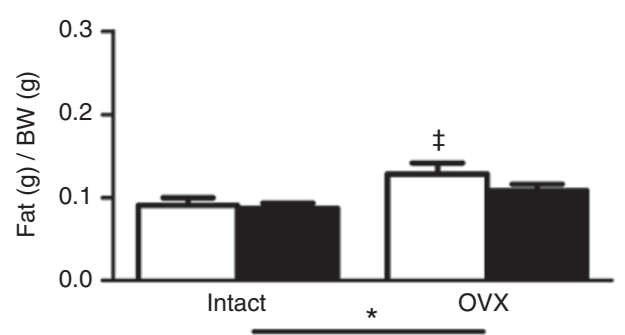

b

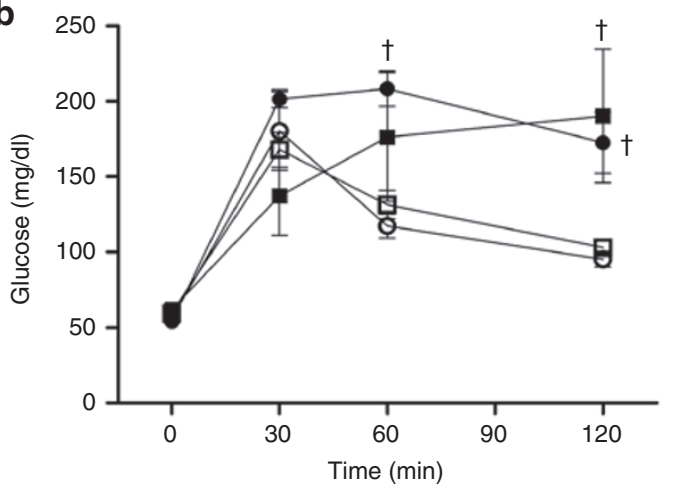

d

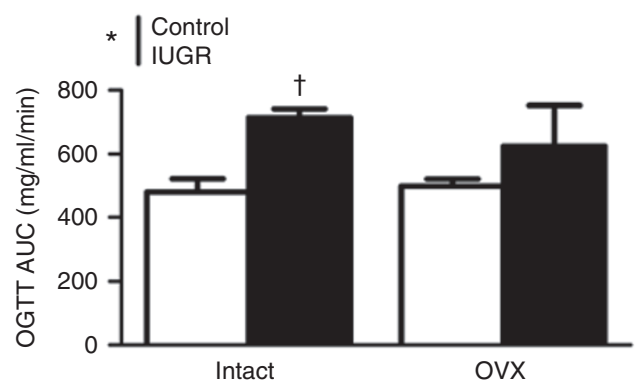

f

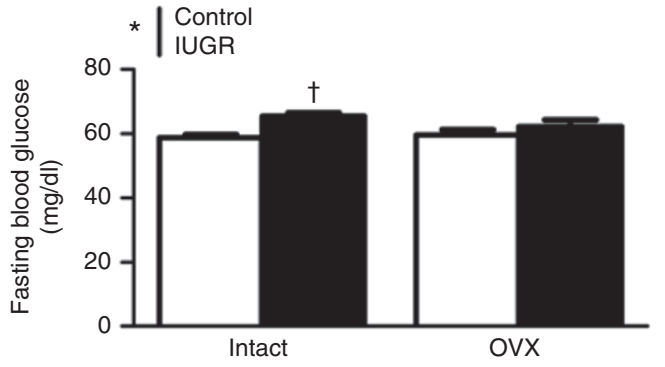

h

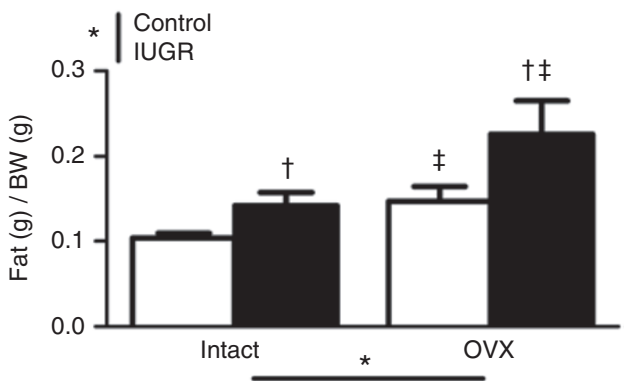

Figure 4. Effect of ovariectomy on glucose homeostasis. Oral glucose tolerance test (OGTT) at 6 (a) and 12 mo of age (b), area under the curve (AUC) at 6 (c) and at 12 mo of age (d), fasting blood glucose levels at $6(\mathbf{e})$ and at 12 mo of age (f), total fat mass per body weight at $6(\mathbf{g})$ and at 12 mo of age (h). ${ }^{*} P$ values next to each label represent the significance in the effect $(P<0.05)$ (intrauterine growth restriction (IUGR) vs. ovariectomy $\left.(O V X)\right)$ using two-way ANOVA; ${ }^{\dagger} P<0.05$ represents IUGR vs. control in intact or OVX; ${ }^{\ddagger} P<0.05$ represents comparison between intact and OVX in similar group using Bonferroni post hoc test, ( $n=8$ per group). For (a) and (b): open circle (control intact), closed circle (IUGR intact), open rectangle (control OVX), and closed rectangle (IUGR OVX). For (c), (d), (e), (f), and (h), white columns represent control and black columns represent IUGR.

is associated with a state of androgen/estrogen imbalance that results from a decrease in estradiol with testosterone levels remaining unchanged or increased (23). Visceral adiposity is significantly associated with higher bioavailable testosterone levels in menopause (12). Higher testosterone levels are also associated with a higher prevalence T2D (24) and hypertension (14) in postmenopausal women implicating a causal role. Recent studies indicate that LBW is associated with earlier age at menopause $(3,4)$. Early menopause is also associated with a greater risk for T2D (15) and the risk for T2D increases with decreasing birth weight in women $(16,25)$. In our study estrus acyclicity in addition to increased adiposity and fasting blood glucose were observed in female growth-restricted rats at 12 mo of age suggesting that IUGR in the rat mimics age-related changes in reproductive health, adiposity and glucose homeostasis in LBW women. Yet, growth-restricted rats exhibited glucose intolerance at 6 mo of age prior to the development of increased adiposity and estrus acyclicity. Hormonal levels 


\section{Articles | Ittapade tal.}

were not determined at 6 mo of age which limits interpretation of our findings. Thus, whether testosterone excess correlates to glucose intolerance in female growth-restricted rats at 6 mo of age is not known. Furthermore, the source(s) of increased testosterone in female growth-restricted rats at 12 mo of age necessitates further investigation but it may involve the ovaries and/or adrenal glands.

Fetal exposure to undernutrition increases the risk of T2D in later life in humans (26). Molecular mechanisms may involve alterations in expression of the glucose transport system. Whether a reduction in GLUT4 protein expression is observed in adipose tissue from LBW women is not reported; however, a reduction in GLUT4 protein expression is noted in adipose (27) and muscle tissue from LBW men (28). In our study, GLUT4 and IR $\beta$ protein expression were not changed in the skeletal muscle of female growth-restricted relative to female control at 12 mo of age, but they were altered in the adipose tissue. Information related to expression of IR $\beta$ in adipose and muscle tissue in LBW women is not known; however, IUGR programs an increase in IR $\beta$ and GLUT4 in adipose tissue of female LBW piglets at birth (17). Whether these changes persist until adulthood and are associated with impaired metabolic health was not studied. Male offspring exposed to maternal protein restriction exhibit a reduction in expression of pancreatic GLUT2, an increase in adiposity and impaired glucose homeostasis in young adulthood (18). Limitations of our study include the lack of investigation into activation of insulin signaling factors as noted by changes in the phosphorylation levels of key proteins in insulin signaling such as IRS-1 and IR and whether glucose uptake in the peripheral tissues is altered following IUGR in the female rat. Yet, these studies indicate that expression of these factors is altered following slow fetal growth and may be contributory factors in the developmental programming of impaired glucose homeostasis.

Hyperglycemia stimulates $\beta$-cell dysfunction and eventual $\beta$-cell death (29). Human and animal studies suggest that estradiol improves glucose homeostasis and is protective against insulin deficiency during the diabetic state (30) Estrogen impacts $\beta$-cell function through its receptors and protects $\beta$-cells against oxidative injury through activation of its estrogen receptor- $\alpha(\mathrm{ER} \alpha)(21)$. ER $\alpha$ stimulates $\beta$-cell insulin biosynthesis and release in the pancreas and ERs are implicated to regulate GLUT4 in muscle and adipose (31) suggesting an important role for ER $\alpha$ in the regulation of glucose homeostasis (32). In our study, female growth-restricted rats at 12 mo of age demonstrated a decrease in protein expression of pancreatic ER $\alpha$ and GLUT2 with an increase in fasting glucose levels. A limitation of our study includes whether $\beta$-cell number was reduced. Impaired glucose homeostasis in male offspring in other models of IUGR induced via placental insufficiency in the rat and sheep is associated with smaller $\beta$-cell number $(33,34)$. However, no difference in pancreatic weight was observed in the female growth-restricted at $12 \mathrm{mo}$ of age. These studies indicate a clear need for more in-depth investigation into how IUGR programs impaired glucose tolerance.
To assess the importance of the shift toward testosterone excess on glucose homeostasis, rats underwent ovariectomy at $10 \mathrm{wk}$ of age followed by study of glucose homeostasis at 6 and 12 mo of age. Glucose intolerance and a significant increase in AUC, fasting blood glucose, and fat mass were observed in ovariectomized control rats at 6 mo of age relative to intact counterparts. Ovariectomy also induced an increase in fat mass and fasting glucose in growth-restricted rats at 6 mo of age that already exhibited glucose intolerance and an increased AUC. Ovariectomy induces an extreme shift in the estradiol to testosterone ratio due to a steep decline in estradiol levels following removal of the ovaries (35). Thus, ovariectomy-induced increases in fasting blood glucose and fat mass in control and growth-restricted rats at 6 mo of age mimicked the metabolic disturbances observed in conjunction with endogenous elevations in testosterone in intact growth-restricted rats at $12 \mathrm{mo}$ of age. These findings would implicate a role for testosterone excess in the etiology of increased fasting glucose and adiposity in female rats. Yet, ovariectomy did not induce an increase in fasting glucose or AUC in control rats at 12 mo of age relative to intact age-matched controls. Furthermore, intact growthrestricted rats at 6 mo of age that exhibited glucose intolerance and increased AUC, retained a normal pattern of estrus cyclicity with no change in fat mass or fasting blood glucose levels relative to age-matched intact controls. Whether testosterone levels were increased in intact growth-restricted rats at 6 mo of age was not examined. However, these findings would suggest that the development of impaired glucose handling requires more than changes in the hormonal milieu in female rats regardless of age.

The pattern of the OGTT curve but not AUC was altered by ovariectomy in growth-restricted rats at 12 mo of age. Insulin release in response to a glucose challenge was not examined in this group. Therefore, the mechanism(s) that contribute to the differential handling of glucose in response to a glucose challenge in ovariectomized growth-restricted rats is unknown. The lack of an effect on glucose homeostasis by ovariectomy in control and growth-restricted rats at 12 mo of age despite the significant ovariectomy-induced increase in fat mass may involve a loss of sensitivity to the effect of testosterone in the female control rats, or resistance to a greater shift toward testosterone excess in female growth-restricted rats that were already acclimated to endogenous increases in testosterone at this age. Thus, whether impaired glucose homeostasis is intrinsic following IUGR in the female rat or whether testosterone plays a contributory role is not well-defined. Tissue-specific differences in insulin signaling and glucose handling may contribute to age-related changes in sensitivity to the hormonal milieu and require further investigation.

To summarize, this study indicated that IUGR programmed an increase in adiposity, early estrous acyclicity and a shift in the estradiol to testosterone ratio at 12 mo of age. Glucose intolerance was observed in growth-restricted rats at 6 mo of age prior to increased adiposity or estrus acyclicity. Whether testosterone excess contributed to the development of glucose intolerance at 6 mo of age is not known. Yet, fasting blood glucose 
levels were not elevated at this age. Increased fasting blood glucose levels did coincide with increased adiposity and a shift toward testosterone excess regardless of whether testosterone excess was due to ovariectomy in control and growth-restricted rats at 6 mo of age or an IUGR-induced endogenous increase in testosterone in intact growth-restricted rats at $12 \mathrm{mo}$ of age. Thus, the etiology of increased fasting glucose may involve the adverse influence of testosterone in the female rat regardless of birth weight. Impaired glucose homeostasis in the growthrestricted rat at 12 mo of age was associated with tissue-specific alterations in protein expression of ER $\alpha$ and downstream mediators of glucose homeostasis in insulin-sensitive tissues suggesting that impaired pancreatic insulin signaling may also serve as a potential mediator in the developmental programming of impaired glucose homeostasis. Limitations for this study include the lack of direct mechanistic investigation into the relationship between IUGR and early estrus acyclicity, measure of sex steroids at $6 \mathrm{mo}$ of age and the correlative findings of increased adiposity, testosterone, and fasting blood glucose levels in female growth-restricted rats at 12 mo of age. However, this study implicates the need for further investigation into estrogen responsiveness at the peripheral and central level in order to elucidate the importance of persistent estrous and the implication of early reproductive senescence on chronic health following growth restriction during fetal life.

\section{Perspectives}

The relative importance of LBW in the transition into menopause is vastly understudied. These findings herald critical implications for LBW women that may postpone childbearing due to professional or socioeconomic reasons and denotes the need for further studies to investigate the mechanisms involved in this programming event. It is well established that birth weight is inversely correlated with risk for hypertension (2) and T2D (25) in postmenopausal women. Thus, this study also highlights the critical need to investigate the underlying mechanisms that contribute to the increased risk for metabolic disease following slow growth during fetal life in order to develop therapeutic strategies to alleviate chronic disease including risk factors for T2D in LBW women.

\section{METHODS}

Expanded methods are included in the Supplementary Methods online.

\section{Animals}

All experimental procedures were conducted in accordance with National Institutes of Health guidelines for the Care and Use of Laboratory Animals with approval by the Animal Care and Use Committee at the University of Mississippi Medical Center. Study groups included: Group 1: Sex steroids and body compositions in female SD rats at 12 mo of age previously analyzed for blood pressure (10); Group 2: Estrous cyclicity and body composition from 5 to 6 mo of age and 11 to 12 mo of age with measure of hormonal levels at 12 mo of age; Group 3: Insulin tolerance test (ITT), OGTT, insulin release in response to an oral glucose challenge in the fasted state and body composition at 6 and 12 mo of age; Group 4: Body composition and harvest of tissues for western blot analysis at 12 mo of age; Group 5: ovariectomy or sham procedure at $10 \mathrm{wk}$ of age followed by body composition and OGTT at 6 and 12 mo of age; measure of uterine weight and serum testosterone at 12 mo of age.
Reduced uterine perfusion in the pregnant rat and the sham procedure was performed as previously described (36).

\section{Ovariectomy in Female Offspring}

Ovariectomy (OVX) was performed at $10 \mathrm{wk}$ of age as previously described (35).

\section{Body Composition}

Total fat mass, total lean mass, and total body water content were determined in conscious animals using an Echo-MRI-700 (Echo Medical Systems, Houston TX).

\section{Glucose Tolerance Test, Glucose-Stimulated Insulin Release in} Response to Glucose Challenge and Insulin Tolerance Test An OGTT and an ITT were performed at 6 and 12 mo of age. Animals were fasted overnight prior to OGTT; the OGTT was initiated with an oral bolus of D-glucose solution ( $5 \mathrm{~g}$ per $\mathrm{kg} \mathrm{BW}$ ) Animals were fasted $3 \mathrm{~h}$ prior to the ITT; ITT initiated with a single injection of insulin $(0.5 \mathrm{U} / \mathrm{kg}$ BW, Lantus insulin glargine (rDNA) origin, i.p). The ITT and OGTT were performed in the same group of animals $1 \mathrm{wk}$ apart at 6 and $12 \mathrm{mo}$ of age.

Homeostasis model assessment (HOMA) index was calculated as fasting insulin $(\mathrm{microU} / \mathrm{ml}) \times$ fasting glucose $(\mathrm{mmol} / \mathrm{l}) / 22.5$ as reported by others $(37,38)$.

Hormone levels were determined using Ultra-Sensitive Estradiol or Testosterone RIA kits, Linco Research, St. Charles, MO; Insulin Ultra-Sensitive Rat Insulin ELISA kit, Crystal Chem, Downers Grove, IL; LH and FSH BioAssay ELISA kits, US Biologicals Life Sciences, Salem, MA.

\section{Estrous Cycle}

The phase of estrous cycle was determined by vaginal cytology as described by Singletary et al. (22).

Western blot analysis was performed as previously described (39).

\section{Statistics}

Graphpad PRISM version 4 (Graph Pad Software, San Diego, CA) was used for all statistical analysis. Differences between groups were evaluated by two-way ANOVA followed by Bonferonroni post-test with IUGR and age or OVX as sources of variation for most of experiments. An unpaired student $t$-test was used for comparison of birth weight, western blot data, hormone levels, and calorimetry measurement data. Differences were considered statistically significant at $P<0.05$. All results are presented as mean \pm SEM.

\section{SUPPLEMENTARY MATERIAL}

Supplementary material is linked to the online version of the paper at http:// www.nature.com/pr

\section{STATEMENT OF FINANCIAL SUPPORT}

This study was supported by National Institutes of Health (NIH) HL074927, HL51971, and P20GM104357; American Heart Association Grant in Aid, GRNT19900004 (BTA); and American Heart Association Post-Doctoral Fellowship Grant, 12POST11980021 and NIH P20GM104357 (SI).

Disclosures: There are no disclosures.

\section{REFERENCES}

1. Bromberger JT, Matthews KA, Kuller LH, Wing RR, Meilahn EN, Plantinga P. Prospective study of the determinants of age at menopause. Am J Epidemiol 1997;145:124-33.

2. Yarde F, Broekmans FJ, van der Pal-de Bruin KM, et al. Prenatal famine, birthweight, reproductive performance and age at menopause: the Dutch hunger winter families study. Hum Reprod 2013;28:3328-36.

3. Steiner AZ, D’Aloisio AA, DeRoo LA, Sandler DP, Baird DD. Association of intrauterine and early-life exposures with age at menopause in the Sister Study. Am J Epidemiol 2010;172:140-8.

4. Tom SE, Cooper R, Kuh D, Guralnik JM, Hardy R, Power C. Fetal environment and early age at natural menopause in a British birth cohort study. Hum Reprod 2010;25:791-8.

5. Bernal AB, Vickers MH, Hampton MB, Poynton RA, Sloboda DM. Maternal undernutrition significantly impacts ovarian follicle number and increases ovarian oxidative stress in adult rat offspring. PLoS One 2010;5:e15558.

6. Guzmán C, Cabrera R, Cárdenas M, Larrea F, Nathanielsz PW, Zambrano E. Protein restriction during fetal and neonatal development in the 


\section{Articles $\mid$ Intapad et al.}

rat alters reproductive function and accelerates reproductive ageing in female progeny. J Physiol 2006;572(Pt 1):97-108.

7. Khorram O, Keen-Rinehart E, Chuang TD, Ross MG, Desai M. Maternal undernutrition induces premature reproductive senescence in adult female rat offspring. Fertil Steril 2015;103:291-8.e2.

8. Chernoff N, Gage MI, Stoker TE, Cooper RL, Gilbert ME, Rogers EH. Reproductive effects of maternal and pre-weaning undernutrition in rat offspring: age at puberty, onset of female reproductive senescence and intergenerational pup growth and viability. Reprod Toxicol 2009;28:489-94.

9. Alexander BT. Placental insufficiency leads to development of hypertension in growth-restricted offspring. Hypertension 2003;41:457-62.

10. Intapad S, Tull FL, Brown AD, et al. Renal denervation abolishes the age-dependent increase in blood pressure in female intrauterine growthrestricted rats at 12 months of age. Hypertension 2013;61:828-34.

11. Durbin PW, Williams MH, Jeung N, Arnold JS. Development of spontaneous mammary tumors over the life-span of the female Charles River (Sprague-Dawley) rat: the influence of ovariectomy, thyroidectomy, and adrenalectomy-ovariectomy. Cancer Res 1966;26:400-11.

12. Janssen I, Powell LH, Jasielec MS, Kazlauskaite R. Covariation of change in bioavailable testosterone and adiposity in midlife women. Obesity (Silver Spring) 2015;23:488-94.

13. Wang L, Szklo M, Folsom AR, Cook NR, Gapstur SM, Ouyang P. Endogenous sex hormones, blood pressure change, and risk of hypertension in postmenopausal women: the Multi-Ethnic Study of Atherosclerosis. Atherosclerosis 2012;224:228-34.

14. Kalyani RR, Franco M, Dobs AS, et al. The association of endogenous sex hormones, adiposity, and insulin resistance with incident diabetes in postmenopausal women. J Clin Endocrinol Metab 2009;94:4127-35.

15. Brand JS, van der Schouw YT, Onland-Moret NC, et al.; InterAct Consortium. Age at menopause, reproductive life span, and type 2 diabetes risk: results from the EPIC-InterAct study. Diabetes Care 2013;36:1012-9.

16. Rich-Edwards JW, Colditz GA, Stampfer MJ, et al. Birthweight and the risk for type 2 diabetes mellitus in adult women. Ann Intern Med 1999;130(4 Pt 1):278-84.

17. Gatford KL, Simmons RA, De Blasio MJ, Robinson JS, Owens JA. Review: Placental programming of postnatal diabetes and impaired insulin action after IUGR. Placenta 2010;31 Suppl:S60-5.

18. Chamson-Reig A, Thyssen SM, Hill DJ, Arany E. Exposure of the pregnant rat to low protein diet causes impaired glucose homeostasis in the young adult offspring by different mechanisms in males and females. Exp Biol Med (Maywood) 2009;234:1425-36.

19. Theys N, Bouckenooghe T, Ahn MT, Remacle C, Reusens B. Maternal low-protein diet alters pancreatic islet mitochondrial function in a sex-specific manner in the adult rat. Am J Physiol Regul Integr Comp Physiol 2009;297:R1516-25.

20. Fernandez-Twinn DS, Wayman A, Ekizoglou S, Martin MS, Hales CN, Ozanne SE. Maternal protein restriction leads to hyperinsulinemia and reduced insulin-signaling protein expression in 21-mo-old female rat offspring. Am J Physiol Regul Integr Comp Physiol 2005;288:R368-73.

21. Le May C, Chu K, Hu M, et al. Estrogens protect pancreatic beta-cells from apoptosis and prevent insulin-deficient diabetes mellitus in mice. Proc Natl Acad Sci USA 2006;103:9232-7.

22. Singletary SJ, Kirsch AJ, Watson J, et al. Lack of correlation of vaginal impedance measurements with hormone levels in the rat. Contemp Top Lab Anim Sci 2005;44:37-42.
23. Burger HG, Dudley EC, Cui J, Dennerstein L, Hopper JL. A prospective longitudinal study of serum testosterone, dehydroepiandrosterone sulfate, and sex hormone-binding globulin levels through the menopause transition. J Clin Endocrinol Metab 2000;85:2832-8.

24. Ding EL, Song Y, Malik VS, Liu S. Sex differences of endogenous sex hormones and risk of type 2 diabetes: a systematic review and meta-analysis. JAMA 2006;295:1288-99.

25. Lawlor DA, Davey Smith G, Ebrahim S. Life course influences on insulin resistance: findings from the British Women's Heart and Health Study. Diabetes Care 2003;26:97-103.

26. de Rooij SR, Painter RC, Roseboom TJ, et al. Glucose tolerance at age 58 and the decline of glucose tolerance in comparison with age 50 in people prenatally exposed to the Dutch famine. Diabetologia 2006;49: 637-43.

27. Ozanne SE, Jensen CB, Tingey KJ, et al. Decreased protein levels of key insulin signalling molecules in adipose tissue from young men with a low birthweight: potential link to increased risk of diabetes? Diabetologia 2006;49:2993-9.

28. Ozanne SE, Jensen CB, Tingey KJ, Storgaard H, Madsbad S, Vaag AA. Low birthweight is associated with specific changes in muscle insulin-signalling protein expression. Diabetologia 2005;48:547-52.

29. Marshak S, Leibowitz G, Bertuzzi F, et al. Impaired beta-cell functions induced by chronic exposure of cultured human pancreatic islets to high glucose. Diabetes 1999;48:1230-6.

30. Mauvais-Jarvis F, Clegg DJ, Hevener AL. The role of estrogens in control of energy balance and glucose homeostasis. Endocr Rev 2013;34:309-38.

31. Ropero AB, Alonso-Magdalena P, Quesada I, Nadal A. The role of estrogen receptors in the control of energy and glucose homeostasis. Steroids 2008;73:874-9.

32. Nadal A, Alonso-Magdalena P, Soriano S, Quesada I, Ropero AB. The pancreatic beta-cell as a target of estrogens and xenoestrogens: Implications for blood glucose homeostasis and diabetes. Mol Cell Endocrinol 2009;304:63-8.

33. Limesand SW, Rozance PJ, Macko AR, Anderson MJ, Kelly AC, Hay WW Jr. Reductions in insulin concentrations and $\beta$-cell mass precede growth restriction in sheep fetuses with placental insufficiency. Am J Physiol Endocrinol Metab 2013;304:E516-23.

34. Simmons RA, Templeton LJ, Gertz SJ. Intrauterine growth retardation leads to the development of type 2 diabetes in the rat. Diabetes 2001;50:2279-86.

35. Ojeda NB, Grigore D, Robertson EB, Alexander BT. Estrogen protects against increased blood pressure in postpubertal female growth restricted offspring. Hypertension 2007;50:679-85.

36. Campbell LR, Pang Y, Ojeda NB, Zheng B, Rhodes PG, Alexander BT. Intracerebral lipopolysaccharide induces neuroinflammatory change and augmented brain injury in growth-restricted neonatal rats. Pediatr Res 2012;71:645-52.

37. Cacho J, Sevillano J, de Castro J, Herrera E, Ramos MP. Validation of simple indexes to assess insulin sensitivity during pregnancy in Wistar and Sprague-Dawley rats. Am J Physiol Endocrinol Metab 2008;295:E1269-76.

38. Duncan MH, Singh BM, Wise PH, Carter G, Alaghband-Zadeh J. A simple measure of insulin resistance. Lancet 1995;346:120-1.

39. Ojeda NB, Hennington BS, Williamson DT, et al. Oxidative stress contributes to sex differences in blood pressure in adult growth-restricted offspring. Hypertension 2012;60:114-22. 